\title{
Efficient Automatic Image Annotation using Weighted Feature Fusion and its Optimization using Genetic Algorithm
}

\author{
Ajimi Ameer \\ Mtech Student \\ Computer and Information Science \\ College Of Engineering,Poonjar
}

\author{
Sree Kumar.K \\ Assist.Professor \\ Computer and Information Science \\ College Of Engineering,Poonjar
}

\begin{abstract}
The conventional way of text based retrieval systems are now being replaced by the visual content based systems in image retrieval. The image content has several dominant characteristics features like texture,color,shape and it is interesting to research the classification of images on contentbasis with these features. These different descriptor can be combined to form a single feature vector. However, in order to get optimum performance and to reduce the feature dimensionality for making system close to human perception, genetic algorithm (GA) based feature selection is used in this paper. Single feature describes image content only from one point of view, which will not give a correct result. Fusing multi- feature similarity score is expected to improve the accuracy of system's retrieval performance. Inorder to assign the fusion weights of multi-feature similarity scores reasonably according to an image, the genetic algorithm is applied.
\end{abstract}

\section{General Terms}

Automatic Image Annotation, Optimization Algorithm

\section{Keywords}

,Genetic Algorithm,Feature vector weights ,Feature fusion, HOG,SURF,HSV.

\section{INTRODUCTION}

Automatic Image Annotation is an annotation technique which assigns tags or captions to a relevant image to increase the efficiency of image retrieval and classification.In conventional automatic image annotation,each feature is assigned equal weights,so that each feature get the same weightage in all images.But in this paper weights are assigned for each features similar to human perception using genetic algorithm which is an optimization technique hich mimics natural selection.

In this method, image representation scheme is achieved using three descriptors, namely, HSV Color Histogram (10 dimensional vector), Histogram of Oriented Gradients (81dimensional vector) and Speeded-Up Robust Features (64 dimensional vector).The weights of this features are obtained using genetic algorithm for each class of images.

\section{RELATED WORKS}

Many techniques and approaches were proposed for optimization in automatically annotating images in various fields. For water quality monitoring, Ni Bin Chang et.al. proposed an Integrated Data Fusion and Machine-learning (IDFM) which is an early warning system for the prediction of microcystin concentrations and distribution by measuring the surface reflectance of the water body using satellite sensors[14]. A fused image is created by the algorithmic fusion of the spectral, temporal, and spatial properties of two or more images. For improved content based retrieval a neural network optimization using genetic algorithm is proposed in [20]. Feature is extracted from an image using Discrete Cosine Transform, extract relevant features using information gain and Gaussian Fuzzy Feed Forward Neural Network algorithm for classification and optimize the momentum and learning rate using Genetic algorithm.

Joey Mark Diaz et.al. utilized genetic algorithm as a method of feature (genes) selection for the support vector machine and artificial neural network to classify lung cancer status of a patient in Lung Cancer Classification study. Genetic algorithm (GA) successfully identified genes that classify patient lung cancer status with notable predictive Performance[16].

Content-based image retrieval (CBIR) of medical images, according to its domain specific image features, is a valuable tool for physicians[17]. The method has three major steps: 1 . Features extracted from the CT images using Coif let wavelets; 2. The features extracted are classified using Support Vector Machine; 3. The parameters of the SVM are optimized using Particle Swarm Optimization (PSO), and modified PSO with a genetic algorithm.

Ji Zhoung et.al [22] proposed a Land Surface temperature retrieval from Moderate-resolution Imaging Spectroradiometer (MODIS) data by integrating regression models and the GA in an arid region. Song and Zhao applied the GA to retrieve component temperature from MODIS data based on a linear spectral mixing model [19]. The GA appears to be a good method for retrieving the Land surface temperature ( LST) from thermal remote sensing images.

Ashok Samal et.al. proposed a method for searching satellite imagery with integrated measures[21].In Retrieval of geospatial imagery, The model structure is determined by a structure learning algorithm with feature selection capabilities. The parameters of the models are optimized through a genetic algorithm. The system is used to classify the landcover wet- land and agricultural zones in a lake-wetland ecosystem in Greece using IKONOS imagery. 


\section{PROPOSED WORK}

A number of features that are used to represent an image can be broadly classified into four major categories, Colour features, texture features, shape features and frequency domain features. Different combinations of these features which are complementing with each other will give better results compared with any single one. Our work is implemented using MATLAB. In this method, image representation scheme is acheived using three descriptors, namely, HSV Colour Histogram (10 dimensional vector), Histogram of Oriented Gradients (81dimensional vector) and Speeded-Up Robust Features (64 dimensional vector).We considered corel dataset of 10 classes with 100 images each,viz;African, Beach, Building, Bus, Elephant, Flower, Dinosaur, Horse, Meal, Mountain.

In this work, implementation is done through three modules, viz; Training phase, Fusion Phase and Annotation phase.

\subsection{Training Phase}

\subsubsection{Feature Extraction}

Feature extraction is the first and vital stage of any classification and annotation problem.Hundred images are trained for each class.

\subsubsection{SURF Feature Extraction}

The proposed architecture uses speeded up robust feature extraction method (SURF) to extract the features of both training and testing images. SURF extraction method is a scale and rotation invariant feature extraction method,which is faster than widely used feature extracting method scale invariant feature transform (SIFT).

\subsubsection{Color Feature Extraction}

For color features HSV color model is used. Hue, Saturation, Value or HSV is a color model that describes colors (hue or tint) in terms of their shade (saturation or amount of gray) and their brightness (value or luminance). The HSVcolor wheel may be depicted as a cone or cylinder. Instead of Value, the color model may use Brightness, making it HSB .

\subsubsection{Texture Feature Extraction}

To determine texture features The texture features is obtained by grey level co-occurrence matrix(GLCM). The texture and shape feature is added to histogram of gradient(HOG) features used for object detection.

\subsubsection{K-Means Clustering}

Now $\mathrm{N}$ number of rows are used to represent $\mathrm{N}$ number of training images. $\mathrm{N}$ feature vectors are clustered in to $\mathrm{k}-$ clusters using Kmeans clustering. Kmeans clustering is a method of cluster analysis which aims to partition $n$ observations into k clusters in which each observation belongs to the cluster with the nearest mean.

\subsection{Fusion Phase}

\subsubsection{Genetic Algorithm for Optimization}

Genetic Algorithm(GA) to assign weights to different features. The genetic algorithm can be used at the level of the feature elements selection, where important features are maintained while ignoring remaining can employ the genetic algorithm. The composite feature vector to be used in image retrieval can be optimized using genetic algorithm. The chromosome of GA was of length as same as that of feature vector. This chromosome has real value between 0 and 1 , randomly generated at each position.This modification helped in bringing the wide range variations in usable percentage of total feature elements. This enabled to evaluate the chromosome's performance of recognition with having even small percentage of elements. This chromosome was multiplied (element wise) with feature vector to be optimized, before using it for recognition.

\subsubsection{Basic Genetic Algorithm}

A genetic algorithm is a problem solving method that uses genetics as its model of problem solving. It's a search technique to find approximate solutions to optimization and searchproblems. Algorithm consists of following phases:

selection, crossover and mutation. The basic genetic algorithm is as follows:

1. [start] Genetic random population of $\mathrm{n}$ chromosomes (suitable solutions for the problem)we selected double vector.

2. [Fitness] Evaluate the fitness function $f(x)$ of each chromosome $\mathrm{x}$ in the solution space or population.

3. [New population] Create a new population by repeating following steps until the new population is complete.

4. [Selection] select two parent chromosomes from a population according to their fitness ( better fitness, means chance to get selected is high).

5. [crossover] Crossover the obtained parents to form new variants of offspring(children)by giving a probability. If no crossover was performed, offspring becomes an exact copy of parents,then we will stuck on a local minima .

6. [Mutation] With a mutation probability, mutate new offspring at each locus (position in chromosome).

7. [Accepting] Place new offspring in the new population.

8. [Replace] Use new generated population for a further sum of the algorithm.

9. [Test] If the end condition is satisfied, stop, and return the

Best solution in the current iterated population.

10. [Loop] Go to step2 for better fitness evaluation.

GAs are good at taking larger, potentially huge, search spaces and exploring them looking for optimal combinations of things and solutions which we might not find in a life time through exploration. GAs are very useful from most of the traditional optimization methods. Genetic algorithms need phenotypic space to be converted into genetic space. So, Genetic algorithms run as coding of different variables as chromosomes. The advantage in coding of variable space is that, it discretizes the solution space even though the function may be continuous. A more striking difference between GAs and most of the traditional optimization method is that GA uses a population of points at one time in contrast to the single point approach by traditional optimization methods,so will not stuck in a single local point minima. This means that GA processes a number of solutions in the solution space at the same time. 


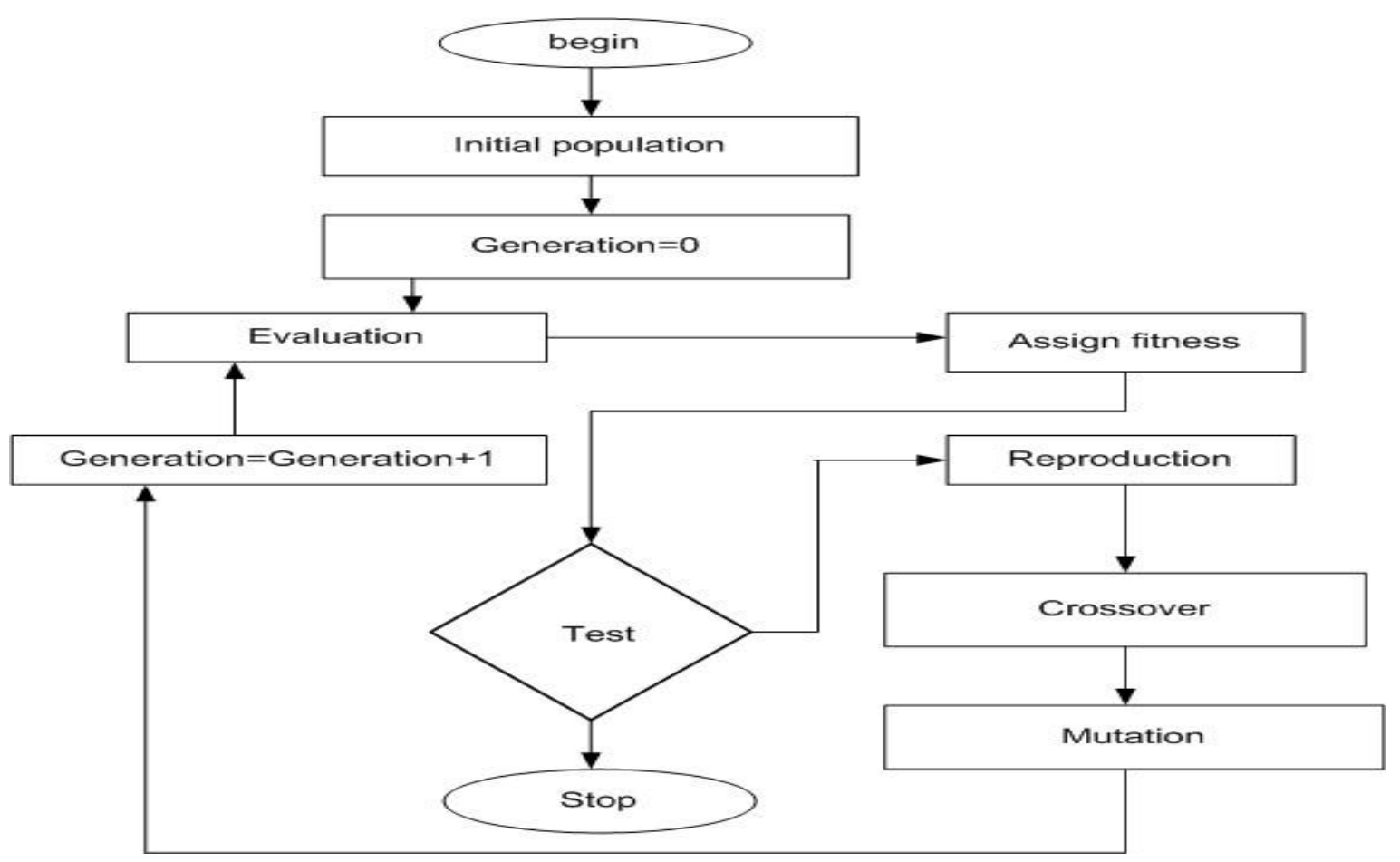

Fig 1: Overview of Genetic Algorithm

\subsection{Annotation Phase}

The process of feature extraction is the same as the feature extraction method described in the training phase.

\subsubsection{Feature Extraction}

Feature extraction is the first and important stage of any classification and annotation problem.

\subsubsection{SURF Feature Extraction}

The proposed architecture uses speeded up robust feature extraction method (SURF) to extract the features of both training and testing images.

3.3.1.2 Color Feature Extraction For color features HSV color model is used.

\subsubsection{Texture Feature Extraction}

A statistical method of examining texture that considers the spatial relationship of pixels is the gray-level co-occurrence matrix (GLCM), also known as the gray-level spatial dependence matrix.

HOG is a feature descriptor that is heavily used in computer and human vision necessitating the object orientation. It is the descriptor that gives the local object appearance and shape within the image described by the intensity of gradient values.

The features should be correctly fused inorder for the best retrieval of images. So,the proposed system should use Genetic Algorithm(GA) to assign weights to different features. The genetic algorithm can be used at the level of the feature elements selection, where important features are given higher weightage while ignoring or reducing the weights of less important features.
Since the physical meanings and representations of different features are dissimilar, their value ranges are dissimilar, so that,similarity scores of different features cannot be compared meaningfully. So,to overcome this problem,we should normalize every feature vectors before finding the weights. Let $\mathrm{Q}$ be the query image. By calculating distances between the query image and images in database, similarity score set $\{\mathrm{Si}\}$ can be gotten, where $\mathrm{i}=1 \ldots \mathrm{N}, \mathrm{N}$ is the number of images in database. Thus, similarity score normalization can be implemented as:

$$
\mathrm{SNi}=\frac{\max \{\mathrm{Si}\}-\min \{\mathrm{Si}\}}{\operatorname{Si}-\min \{\mathrm{Si}\}}
$$

Also,in this work the weights are obtained from genetic algorithm by comparing the similarity measure of some known images of each class and taking the average of feature vectors of each class and then taking the best image that gave the best score.Then the feature vector of this image is given as input to genetic algorithm. The results of multi-feature similarity scores is calculated using the following equation:

$$
\begin{aligned}
\operatorname{Sim}(\mathrm{C}, \mathrm{K})= & \sqrt{ }\left[\left(\sum \text { Csurf }-\sum \text { Ksurf }\right)^{2}\right]+\sqrt{ }\left[\left(\sum \text { Chog }-\sum \text { Khog }\right)^{2}\right]+ \\
& \sqrt{ }\left[\left(\sum \text { Chsv }-\sum \text { Khsv }\right)^{2}\right]
\end{aligned}
$$

Where $\mathbf{C}$ is the average feature vector representing each class and $\mathbf{K}$ is the feature vector of known images of each class.In this work,we took five known images for each class And weights of features find out using the fitness function as:

SFi = SNsurf(i). Wsurf + SNhog(i). Whog +SNhsv(i). Whsv

$$
\text { Wsurf+ Whog +Whsv }
$$


SFi -fused similarity score, SNsurf(i)- normalized surf feature similarity score, SNhog(i)- normalized hog feature similarity score, , SNhsv(i)- normalized hsv feature similarity score, Wsurf -Weight of surf feature similarity score, Whog-Weight of hog feature similarity score, Whsv -Weight of hsv feature similarity score.
In this work, Wsurf+ Whog + Whsv $=1$

Crossover probability taken as $\mathbf{0 . 8}$ and mutativn (4) mutationadaptfeasible since constraints are used.

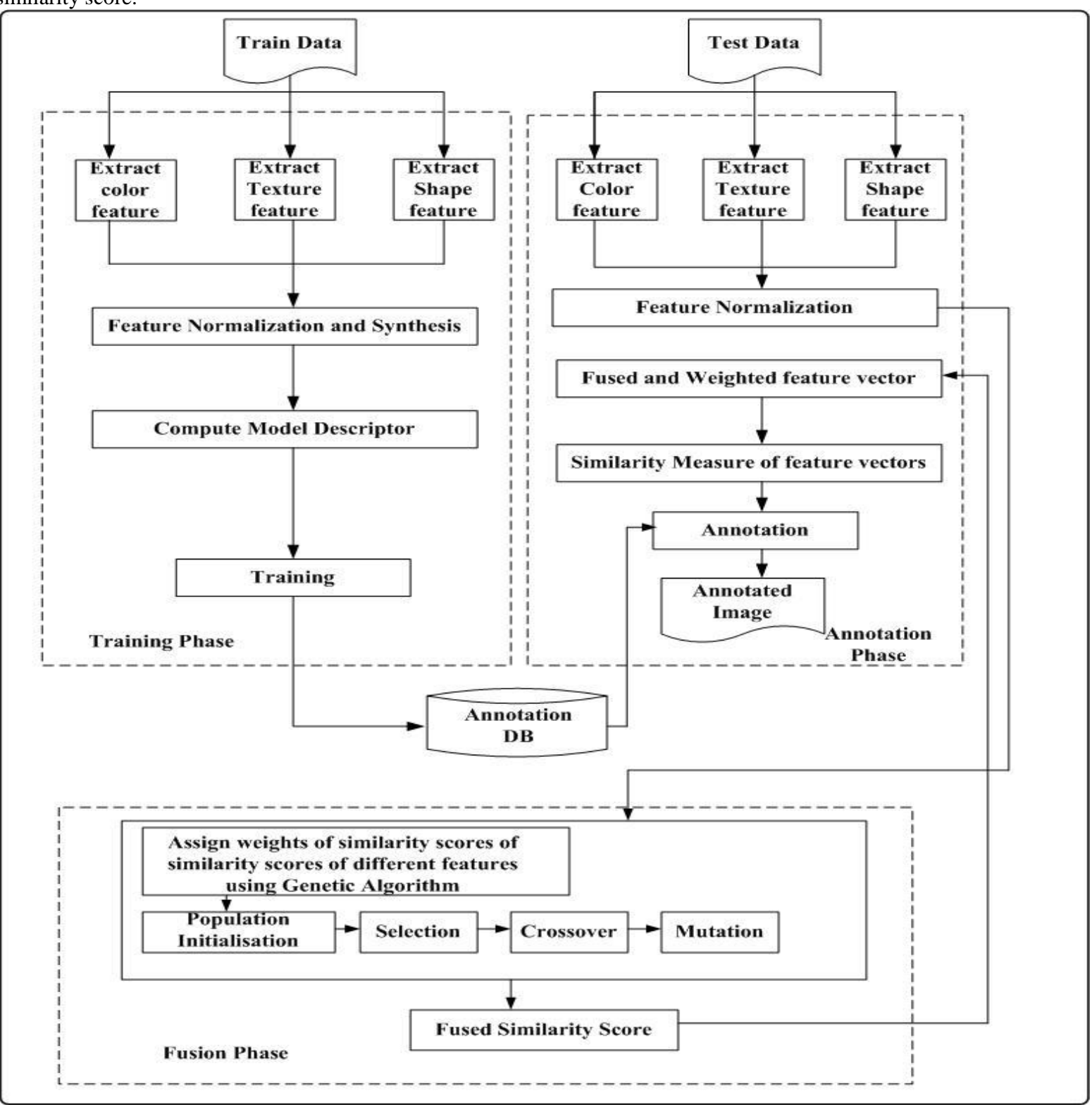

Fig 2: Proposed Architecture

\section{CONCLUSION}

The current work is aimed at GA based approach that can be evolved from the randomly generated solution for the selection of appropriate feature elements from combined feature vector that describes different feature descriptor of the image such as color,texture,shape. So,in this work accuracy of retrieved images increased because weights of different features for each class of image is assigned properly using GA. There is a lot of scope for improving the proposed system including making GA interactive to the user which gives user's freedom for selecting the appropriate images for next generation,additionally improving the constraints and probability of selection and crossover accordingly.

\section{ACKNOWLEDGMENTS}

First and the foremost, I give thanks to Almighty God who gave me the inner strength, resource and ability to complete my seminar successfully, without which all my efforts would have been in vain.I would also like to thank,our HOD Ms. Sindhu L. for her valuable help and support. I am highly obliged to her for all the valuable suggestions and guidance she provided. I express my sincere gratitude to my project guide Mr.SreeKumar .K for his guidance and valuable assistance. 


\section{REFERENCES}

[1] Chandrashekhar G.Patil, Dr.Mahesh

T.Kolte2, Dr.Devendra S.Chaudhari, Fusion at Features Level in CBIR System using Genetic Algorithm,2013

[2] Sapthagiri.k, Manickam , An Efficient Image Retrieval Based on Color, Texture (GLCM \& CCM), features, and Genetic-Algorithm,2013

[3] K. APARNA, Retrieval of Digital Images Based On Multi-Feature Similarity Using Genetic Algorithm, 2013

[4] Ruaa Mohammed Hamza, Dr. Tawfiq A. Al-Assadi, Genetic Algorithm to find optimal GLCM features,2012.

[5] Shafimirza,Dr.J.Apparao, Retrieval Of Digital Images Using Texture Feature With Advanced Genetic Algorithm,2012

[6] S.Sreenivas Rao , Mr. K.Ravi Kumar, Dr. G. Lavanya Devi, Texture Based Image retrieval using Human interactive Genetic Algorithm,2013

[7] S.Gopalakrishnan, Dr. P. Aruna, Retrieval of images based on low level features using genetic algorithm,2014

[8] K.Kalaiyarasi ,A.Kabilar M. Image Retreival Based On Colour, Texture and Shape Analysis Using Genetic Algorithm,2014

[9] P.Kishore Kumar, M.Radhika, Using Genetic Algorithm Image Retrieval Based on Multi- Feature Similarity Score Fusion,2014

[10] Dr.Mahesh.T.Kolte, Chandrashekhar, Improvement in Performance of CBIR by using fusion and Evolutionary Computation, 2014

[11] Anita Nanasaheb Ligade, Manisha R. Patil, Optimized content based image retrieval using genetic algorithm with relevance feedback technique,2013

[12] Miguel Arevalillo-Herraez, Francesc J. Ferri, Salvador Moreno-Picot, An interactive evolutionary approach for content based image retrieval,2013

[13] M. Venkat Dass, Mohammed Rahmath Ali, Mohammed Mahmood Ali, Image Retrieval Using Interactive Genetic Algorithm,2014
[14] Ni-Bin Chang and Benjamin Vannah, Compartive Data Fusion between Genetic Programing and Nueral Network Models for Remote Sensing Images of Water QualityMonitoring, 2014

[15] Itedal Sabri Hashim Bahia, Using Artificial Neural Network Modeling in Forecasting Revenue: Case Study in National Insurance Company/Iraq, 2013

[16] Joey Mark Diaz, Raymond Christopher Pinon, Geoffrey Solano, Lung Cancer Classification Using Genetic Algorithm to Optimize Prediction Models,2013

[17] Ms.N.T.Renukadevi and Dr.P.Thangaraj , Performance analysis of optimization techniques for medical image retrieval,2014

[18] Miguel Arevalillo-Herr'aez, Francesc J. Ferri, Salvador Moreno-Picot, An interactive evolutionary approach for content based image retrieval,2013

[19] Song, X.N.; Zhao, Y.S. Study on component temperatures inversion using satellite remotely sensed data. Int. J. Remote Sens. 2007.

[20] C.Ramesh babu durai, V.Duraisamy, C.Vinothkumar,Improved Content Based Image Retrieval Using Neural Network Optimization with Genetic Algorithm 2012

[21] AshokSamala,,SanjivBhatiab,PrasanthVadlamania, DavidMarxc, Searching satellite imagery with integrated measures, 2009

[22] Ji Zhou, Xu Zhang, Wenfeng Zhan and Huailan Zhang ,Land Surface Temperature Retrieval from MODIS Data by Integrating Regression Models and the Genetic Algorithm in an Arid Region ,2014

[23] Dengsheng Zhang, Aylwin Wong, Maria Indrawan, Guojun Lu, Content-based Image Retrieval Using Gabor Texture Features.

[24] Chandrika L, Implementation Image Retrieval and Classification with SURF Technique, 2014

[25] MS. R.Janani,Sebhakumar.P ,An Improved CBIR Method Using Color and Texture Properties with Relevance Feedback, 2014. 